\title{
NON-CONTROLLED CLOSE-RANGE PHOTOGRAMMETRY METHOD BASED ON PHOTOMODELER SCANNER
}

\author{
HUANG Chang-cheng ${ }^{1}$, LI Yun-jian ${ }^{2}$, TANG Shi-hua ${ }^{1}$, ZHANG yan ${ }^{1}$, XIAO yan ${ }^{1}$ \\ ${ }^{1}$ College of Geomatics and Geoinformation Guilin University of Technology, Guilin, China - gut@glut.edu.cn \\ ${ }^{2}$ Guangxi Zhuang Autonomous Region Surveying and Mapping Geographic Information Product Quality Inspection Station, \\ Nanning, China
}

KEY WORDS: Close-range Photogrammetry; PhotoModeler Scanner; USB Camera; Non-controlled; Detail Survey

\begin{abstract}
:
Aiming at the high demand of close-range photogrammetry for the object control points and the inconvenient carrying of digital camera to take photogrammetry in the field, a method of detail survey based on PhotoModeler Scanner software is proposed. USB camera combines with the centering rod, USB camera captures image data, Total station obtains the coordinates of the centering rod, the coordinate of projective center is calculated by using the coordinate of the centering rod, PhotoModeler Scanner software processes image data and coordinate data. Ultimately, image stereo measurement of the no object control point was realized. The experimental results showed that the error of the plane position of the solved target point was less than $10 \mathrm{~cm}$ at a shooting distance of about $10 \mathrm{~m}$, and it met the accuracy requirements of detail survey. Therefore, the method can reduce the workload of field detail survey, reduce the cost and volume of the photographic equipment, and has certain application value.
\end{abstract}

\section{INTRODUCTION}

Close-range photogrammetry has the characteristics of instantaneous acquisition of rich information of the measured object, non-contact measurement, non-injury to the measured object, easy storage of information, reusable information, high measurement accuracy, low cost, fast speed, low labor intensity in field, etc. and Close-range photogrammetry is widely used in various fields [1-2]. The application of close-range photogrammetry in detail survey can complement the disadvantages of traditional measurement methods of detail survey and improve the efficiency [3-4].Zhang zuxun et al. proposed a photo total station system(PTSS), which was an integration of mature digital photogrammetry technology and high-precision engineering measuring instrument (total station) [5].Yang liu designed a photographic theodolite system by fastening a non-measuring digital camera with a theodolite telescope, which can reach the accuracy level of medium-level close-range photogrammetry (point position error was less than $5 \mathrm{~mm}$ ) [6].He yingpeng had designed a photographic total station system that combined ordinary digital camera and total station to realize close-range photogrammetry without control points. The photographic total station system can quickly and efficiently obtain the point cloud model of buildings [7]. Gao shen et al. had designed a detail survey method of GPS RTK and ordinary camera independent absolute orientation. The method realized the close-range photogrammetry without ground control points, and the accuracy of detail survey points was better than $10 \mathrm{~cm}[8]$.Wang xinyu et al. directly combined the theodolite and camera to realize automatic measurement of visual guidance theodolite, and conducted observation through image sensor instead of human eyes aiming at the target. The combiner he designed had a high degree of automation, but the production is difficult and their cost is high [9].

Fixed connection of non-measuring digital camera with theodolite, total station, GPS RTK or other devices would make measuring equipment cumbersome and be not conducive to field measurement [10]. Traditional photogrammetry can achieve high precision measurement, but it needs to arrange the object control point. Traditional photogrammetry is not suitable

First author: HUANG Chang-cheng - E-mail: 312263588@qq.com. Corresponding author: LI Yun-jian - E-mail: 53521295@qq.com 
for the measurement which requires simple and quick operation and lower precision. In this paper, USB camera is adopted to shoot images. Although the imaging effect is not as good as that of digital camera, the weight and volume of the device are reduced, making field measurement more convenient. By using the characteristics of PhotoModeler Scanner, a measurement method is designed, which gets rid of the dependence on object control points in photogrammetry. The method can meet the precision requirements of 1:500 digital mapping within a certain distance.

\section{COMPOSITION AND DESIGN IDEAS OF EQUIPMENT}

The purpose of the design is to use the three-dimensional coordinates of three known shooting sites as the basic data in the case of no object control point coordinates, and to build a digital model in PhotoModeler Scanner software to realize the three-dimensional coordinate measurement of the target points to be measured. For this purpose, a set of close-range photogrammetry equipment is designed, including a centering rod, a prism, a USB camera, an angle sensor, a smartphone, an OTG data cable. A total station observes the prism mounted on the centering rod, and the prism and centering rod are to obtain the coordinates of the shooting sites, and then the coordinates of the shooting sites will be converted into the coordinates of the projective center in computer. The USB camera is fixed on the centering rod, and the projective center light of the camera is perpendicular to the centering rod. The angle sensor is mounted on the camera to measure the azimuth angle of the main optical axis of the camera. The smartphone is mainly used for manipulate USB cameras and angle sensors and stores data. The OTG data cable is used for data transmission between smartphone and USB camera and angle sensor. The close-range photogrammetry equipment is shown in figure 1 .

General design idea:

First, the centering rod is placed on an appropriate point, which is called the shooting site. The USB camera aims at some target points to shoot an image, and the three-dimensional coordinates $(x, y, z)$ of the shooting sites are measured by the total station. In this way, image data and location data are collected at three locations that are not collinear.
Secondly, the vertical height from the bottom of the centering rod to the projective center is $h$, the plane distance from the centering rod to the projective center is $d$, and the azimuth of the centering rod to the projective center is $\theta$. The three-dimensional coordinates of the projective center point are as follows: $(x+d \sin \theta, y+d \cos \theta, z+h)$.

Finally, we can complete the absolute orientation of the image and the image stereo measurement by using the calculated coordinates of the three projective center points.

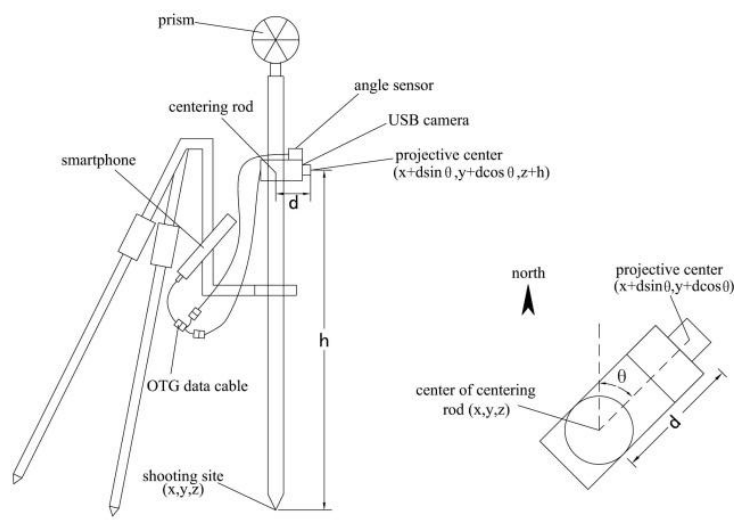

Figure 1. Schematic diagram of equipment structure

\section{THE BASIC THEORY OF CLOSE-RANGE PHOTOGRAMMETRY}

\subsection{Calibration of Cameras}

Before using the ordinary digital camera to carry on the close-range photogrammetry, it must carry on the strict calibrating, in order to restore the relative geometry relation between the projective center and the image [11]. USB camera must be calibrated before taking images. The main content of the camera calibration is to obtain the basic information of the camera, lens distortion parameters and the inside azimuth element of the camera. In this paper, a camera calibration method based on 2D targets proposed by Mr. Zhang zhengyou is adopted. This method only needs the relationship between corresponding points in multiple images to achieve camera calibration, which is simple and flexible, and its accuracy can meet the requirements of this experiment [12-13].

The calibration steps of USB camera are as follows. Frist, The main optical axis of photography keep $45^{\circ}$ from the indoor 
horizontal calibration board. Second, USB camera shoots 4 banner images from 4 directions, and then rotates $90^{\circ}$ with the main optical axis of the lens to shoot 4 longitudinal images. Finally, eight images are imported into PhotoModeler Scanner software for camera calibration [14]. The images and part of the processing process are shown in figure 2 .

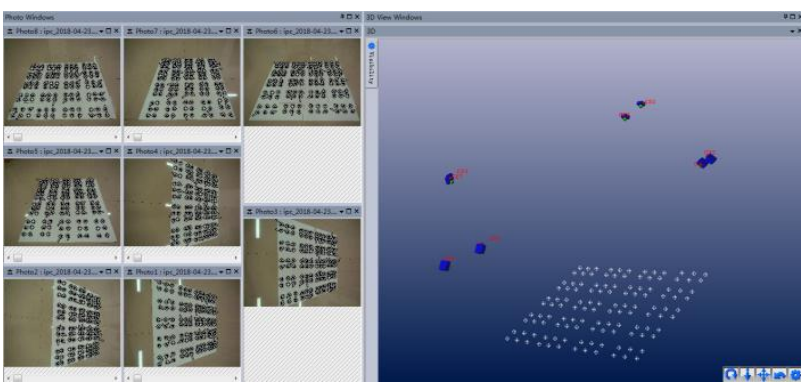

Figure 2. Processing of camera calibration

The quality of camera calibration can be evaluated by image point residuals. It can be seen from the effect diagram of image point residuals magnified by 1000 times to the left of figure 2 that the image point residuals are small and have no fixed direction, indicating that the camera calibration method is stable, reliable and high precision. The report of calibration showed that the focal length of USB camera was $4.893054 \mathrm{~mm}$, and the image format size was $3264 \times 2448$ pixels, and the coordinates of the main image point were $(3.3029,2.2827) \mathrm{mm}$ At the same time, the data of lens distortion and accuracy evaluation were showed, which showed that the accuracy of the calibration results met the requirements.

\subsection{Relative Orientation and Absolute Orientation of Images}

PhotoModeler Scanner software adopts the analytical method of relative orientation. The same object is photographed at two shooting sites and the two images make up a stereo image pair. All the eponymous rays intersect at an object point, which is the internal geometric relationship of the stereo image pair. The eponymous rays intersect in pairs, that is, each pair of eponymous rays is coplanar with the photographic baseline. Let $S_{1}$ and $S_{2}$ be the projective center of the left image and right image, $a_{1}$ and $a_{2}$ are a pair of namesake points. The coplanar condition of relative orientation is that the mixed product of photographic baseline $b=\left(\boldsymbol{S}_{1} \boldsymbol{S}_{2}\right)$ and a pair of eponymous rays $R_{1}=S_{1} a_{1}$ and $R_{2}=S_{2} a_{2}$ is equal to zero, in other words, the formula is $\boldsymbol{b} \cdot\left(\boldsymbol{R}_{\mathbf{1}} \times \mathbf{R}_{\mathbf{2}}\right)=0$. The relative orientation of the continuous image pair is based on the left image, and the relative orientation elements of the right image relative to the left image are obtained. Now, the image-space coordinate system of the left image is taken as the image-space auxiliary coordinate system. Then, the relative orientation elements of the left and right images are:

Left image: $X_{S 1}=0, Y_{S 1}=0, Z_{S 1}=0, \varphi_{1}=0, \omega_{1}=0$, $\kappa_{1}=0$

Right mages: $X_{S 2}=b_{x}, Y_{S 2}=b_{y}, Z_{S 2}=b_{z}, \varphi, \omega, \kappa$

Since $b_{x}$ only affects the size of the model established after relative orientation, and does not affect the establishment of the model, there are only five elements to be solved with respect to relative orientation: $b_{y}, b_{z}, \varphi, \omega, \kappa$. These five elements are called continuous images of relatively oriented elements. $Q$ value is the upper and lower parallax of model points in relative orientation. In stereo image pairs, an equation $Q$ can be listed for each pair of eponymous image point coordinates measured and inner azimuth elements are known. The upper and lower parallax $Q=0$ marks the completion of relative orientation, and there are 5 unknown relative orientation elements. Therefore, at least 5 pairs of eponymous image points should be measured and 5 equations of $Q$ listed. When there are redundant observed values, that is, the number of equations is more than the number of unknowns, the error equation should be listed and calculated according to the adjustment of least square principle. In photogrammetry, the relative orientation process does not need the coordinates of control points, but only needs the image coordinates of 6 pairs of obvious image eponymous points in the overlapping range to solve the relative orientation elements. Images are imported into PhotoModeler Scanner, 6 pairs of eponymous points are selected to complete the relative orientation of stereo image pairs, and a three-dimensional model including the projective center point is established. This model is only similar to the original object, but its size, orientation and absolute position are not restored.

Absolute orientation refers to the process of transforming the photogrammetric coordinate system of three-dimensional model into the target coordinate system. The absolute orientation of image requires solving seven absolute orientating elements. The seven absolute orientating elements are $\Phi, \Omega$, $K, \Delta X, \Delta Y, \Delta Z, \lambda$. The absolute orientation is called "similarity transformation of three-dimensional space with 
different origin" in mathematics. The transformation formula is as follows:

$$
\left(\begin{array}{l}
X_{m} \\
Y_{m} \\
Z_{m}
\end{array}\right)=\lambda\left(\begin{array}{lll}
a_{1} & a_{2} & a_{3} \\
a_{1} & a_{2} & a_{3} \\
a_{1} & a_{2} & a_{3}
\end{array}\right)\left(\begin{array}{l}
X_{P} \\
Y_{P} \\
Z_{P}
\end{array}\right)+\left(\begin{array}{l}
\Delta X \\
\Delta Y \\
\Delta Z
\end{array}\right)
$$

In formula (1), $\left(X_{m}, Y_{m}, Z_{m}\right)$ is the coordinate of object point in the target coordinate system. $\lambda$ is the model scaling factor. $a_{i}, b_{i}, c_{i}(i=1,2,3)$ are directional cosines of $\Phi, \Omega$, $K .\left(X_{p}, Y_{p}, Z_{p}\right)$ is the coordinate of object point in the photogrammetric coordinate system. $\Delta X, \Delta Y, \Delta Z$ are the quantity of translation of the coordinate origin

Formula (1) is a nonlinear function, which is linearized according to the first term of Taylor series formula, and then solved by iterative method. The formula has seven unknowns, and we need at least seven equations to solve these seven unknowns. In close-range photogrammetry, if the ground three-dimensional coordinates of three projective center points are known, nine equations can be listed, so there are redundant observation values, which can be solved by least square adjustment. The three-dimensional coordinates of the three projective center points were inputted in the software PhotoModeler Scanner, to solve the absolute orientation elements, and complete the absolute orientation. Photogrammetric coordinates of all model points can be transformed into ground measurement coordinates.

\section{EXPERIMENTAL ANALYSIS}

A simple hardware system of close-range photogrammetry consists of a USB camera, a centering rod, a prism, an angle sensor, a smartphone and an OTG data line. In addition, a total station is used for measure the coordinates of the shooting sites. The USB camera model is RER-USB8MP02G and it has 8 million pixels. The angle sensor model is JY901 and measurement error of the angle sensor is 1 degree. The simple hardware system of close-range photogrammetry is shown in figure 3 .

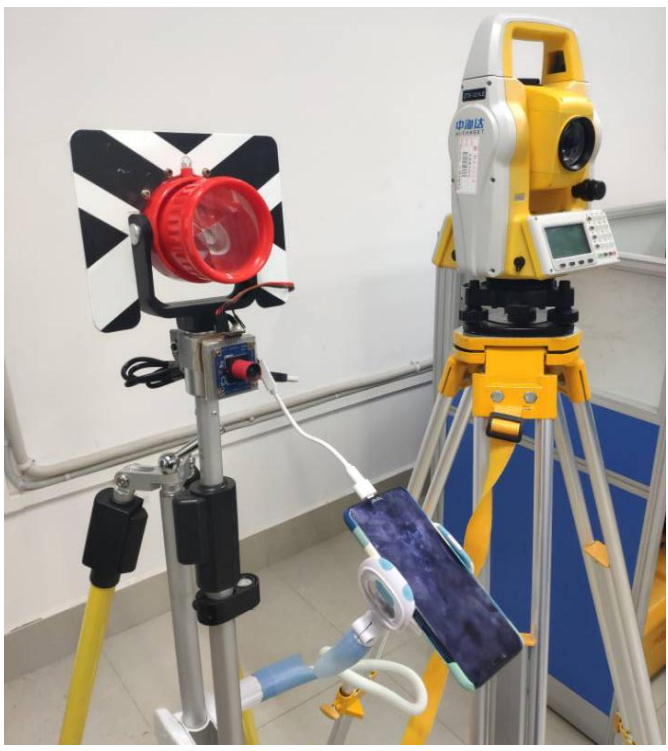

Figure 3. Simple hardware system for close - range photogrammetry

After assembling the hardware system of close-range photogrammetry, the plane distance from the center of centering rod to the projective center was fixed. This distance was measured several times with the total station and averaged, and the average of this distance was denoted by $d$, and the value of $d$ was $0.058 \mathrm{~m}$. The vertical height from the bottom of the centering rod to the projective center was denoted by $h$, and the value of $h$ could be calculated by observing the scale of the centering rod. The angle sensor was used to measure the azimuth of photographic center light, and this azimuth was denoted by $\theta$. Values of $\theta$ can be displayed and recorded by the phone when it was connected to the angle sensor. When the phone was connected to the USB camera, images from the USB camera were displayed on the phone, and the phone stored images from the USB camera. The three-dimensional coordinates $(x, y, z)$ of the shooting sites are measured by the total station. The three-dimensional coordinates of the projective center point were $(x+d \sin \theta, y+d \cos \theta, z+h)$ after deductive calculation. The collected three images would be imported into PhotoModeler Scanner, 6 pairs of eponymous points would be selected manually in the three images, and relative orientation of the three images would be conducted to establish a three-dimensional stereo model. The coordinates of the three photography center points were inputted into PhotoModeler Scanner to complete the absolute orientation of the three images. In the end, any target point to be measured was selected for stereo measurement. 
The first experimental site was an indoor three-dimensional control field, in which posts with different spatial distances were placed, and targets were pasted on the posts at a certain distance. The second experimental site was the roof of a teaching building, and some reflectors were attached to the surfaces of stone benches or pillars. In the two sites, the pictures of the target point would be shot at three locations that were not collinear, and the coordinates of the shooting site would be measured with the total station. The coordinates of the shooting location were used as the basic coordinate data. The total station measured the coordinates of the target points, and the results were used as check data.

In first experiment, 20 target points on the posts were selected as the observation target points, which were divided into three layers. The distance between the points was 1 to 2 meters, and the distances between the shooting sites and the target points were 5 to 8 meters. The experiment process in the indoor control field was shown in figure 4.

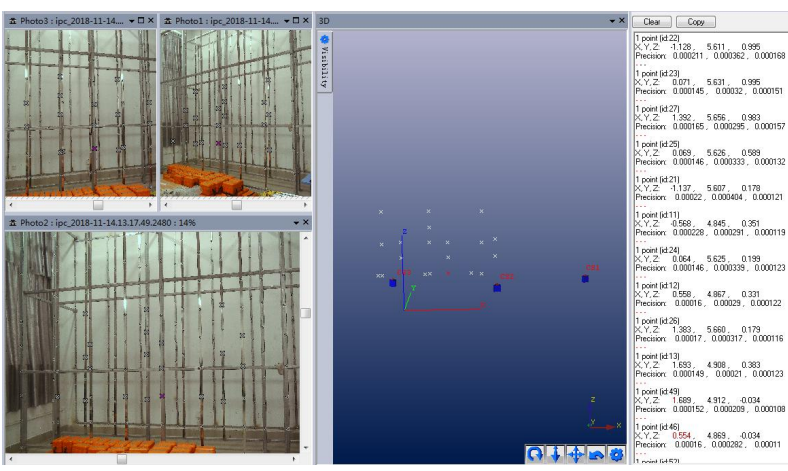

Figure 4. Experiment in Indoor Control Field

In figure 4, the left part showed three images, the middle part showed the stereo model of selected target points and camera positions, and the right part showed measurement results. The results of stereo measurement were exported and compared with the three-dimensional coordinates of the corresponding points measured by the total station. The difference between photogrammetric coordinates and total station coordinates was calculated. The coordinate errors are shown in table 1 .

Table 1. Accuracy Analysis of Indoor Ground Point Coordinates

\begin{tabular}{|c|c|c|c|c|c|c|c|c|c|}
\hline \multirow{2}{*}{$\begin{array}{c}\text { Target } \\
\text { Number }\end{array}$} & \multicolumn{3}{|c|}{ Total Station Coordinates } & \multicolumn{3}{|c|}{ Photogrammetric Coordinates } & \multicolumn{3}{|c|}{ Error Analysis } \\
\hline & $X$ & $\mathrm{Y}$ & $\mathrm{Z}$ & $\mathrm{X}$ & $\mathrm{Y}$ & $\mathrm{Z}$ & $\mathrm{dX}$ & $d Y$ & $\mathrm{dZ}$ \\
\hline 1 & -1.138 & 4.838 & -0.454 & -1.114 & 4.850 & -0.454 & -0.023 & -0.012 & 0.000 \\
\hline 2 & -0.005 & 4.868 & -0.441 & 0.019 & 4.879 & -0.443 & -0.025 & -0.012 & 0.002 \\
\hline 3 & 1.112 & 4.900 & -0.440 & 1.138 & 4.911 & -0.444 & -0.026 & -0.011 & 0.004 \\
\hline 4 & -0.568 & 4.845 & 0.351 & -0.543 & 4.859 & 0.350 & -0.025 & -0.013 & 0.000 \\
\hline 5 & 0.558 & 4.867 & 0.331 & 0.584 & 4.880 & 0.329 & -0.026 & -0.013 & 0.002 \\
\hline 6 & 1.693 & 4.908 & 0.383 & 1.721 & 4.921 & 0.379 & -0.028 & -0.013 & 0.003 \\
\hline 7 & -1.137 & 5.607 & 0.178 & -1.113 & 5.621 & 0.177 & -0.024 & -0.014 & 0.002 \\
\hline 8 & -1.128 & 5.611 & 0.995 & -1.103 & 5.627 & 0.994 & -0.026 & -0.016 & 0.001 \\
\hline 9 & 0.071 & 5.631 & 0.995 & 0.098 & 5.646 & 0.993 & -0.027 & -0.016 & 0.002 \\
\hline 10 & 0.064 & 5.625 & 0.199 & 0.089 & 5.639 & 0.195 & -0.026 & -0.014 & 0.003 \\
\hline 11 & 0.069 & 5.626 & 0.589 & 0.096 & 5.641 & 0.586 & -0.026 & -0.015 & 0.003 \\
\hline 12 & 1.383 & 5.660 & 0.179 & 1.410 & 5.673 & 0.174 & -0.027 & -0.013 & 0.005 \\
\hline 13 & 1.392 & 5.656 & 0.983 & 1.421 & 5.671 & 0.979 & -0.028 & -0.015 & 0.004 \\
\hline 14 & -0.570 & 4.848 & -0.027 & -0.546 & 4.861 & -0.028 & -0.025 & -0.013 & 0.001 \\
\hline 15 & 0.554 & 4.869 & -0.034 & 0.580 & 4.882 & -0.036 & -0.026 & -0.012 & 0.002 \\
\hline 16 & 1.689 & 4.912 & -0.034 & 1.716 & 4.924 & -0.038 & -0.027 & -0.012 & 0.004 \\
\hline 17 & 0.551 & 4.872 & -0.428 & 0.576 & 4.883 & -0.431 & -0.025 & -0.011 & 0.003 \\
\hline 18 & -1.148 & 5.601 & -0.627 & -1.125 & 5.614 & -0.629 & -0.023 & -0.012 & 0.002 \\
\hline 19 & 0.053 & 5.622 & -0.598 & 0.077 & 5.634 & -0.602 & -0.025 & -0.012 & 0.004 \\
\hline 20 & 1.374 & 5.664 & -0.628 & 1.400 & 5.675 & -0.634 & -0.026 & -0.012 & 0.006 \\
\hline RMSE & & & & & & & 0.0270 & 0.0137 & 0.0030 \\
\hline
\end{tabular}


As could be seen from table 1 , the standard deviations of $d X, d Y$ and $d Z$ were $0.0270 \mathrm{~m}, 0.0137 \mathrm{~m}$ and $0.0030 \mathrm{~m}$ respectively. Horizontal position error was $0.0301 \mathrm{~m}$ after calculation. In 1:500 digital mapping, the horizontal position error was required to be less than or equal to $10 \mathrm{~cm}$. Therefore, the results of this photogrammetry can meet the requirements of 1:500 digital mapping.

In second experiment, 10 reflectors were attached to the surfaces of stone benches or pillars on the roof of a teaching building. The distances between the shooting sites and the reflectors were 8 to 12 meters. The method of photogrammetry is the same as that adopted in first experiment. The process of outdoor photogrammetry experiment is shown in figure 5 .

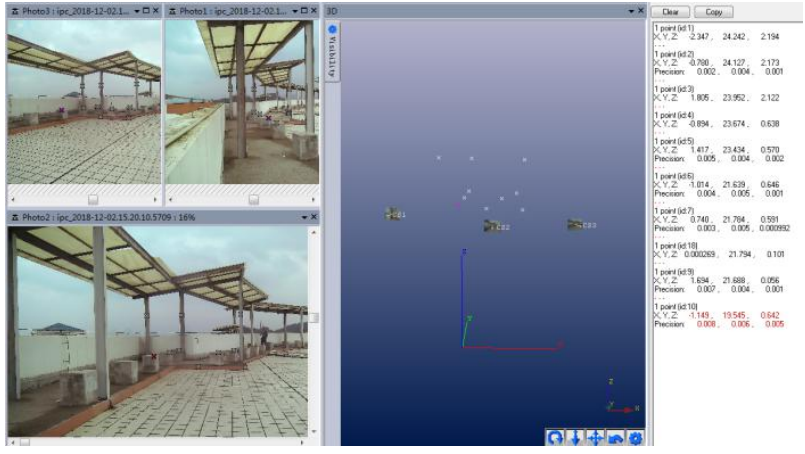

Figure 5. Outdoor Photogrammetry Experiment

In the same way, the data of stereo measurement are exported and compared with the three-dimensional coordinates of the corresponding points measured by the total station. The accuracy analysis of outdoor target point is shown in table 2 .

\begin{tabular}{|c|c|c|c|c|c|c|c|c|c|}
\hline \multirow{2}{*}{$\begin{array}{c}\text { Target } \\
\text { Number }\end{array}$} & \multicolumn{3}{|c|}{ Total Station Coordinates } & \multicolumn{3}{|c|}{ Photogrammetric Coordinates } & \multicolumn{3}{|c|}{ Error Analysis } \\
\hline & $\mathrm{X}$ & $\mathrm{Y}$ & $\mathrm{Z}$ & $\mathrm{X}$ & $\mathrm{Y}$ & $\mathrm{Z}$ & $\mathrm{dX}$ & $\mathrm{dY}$ & $\mathrm{dZ}$ \\
\hline 1 & -2.347 & 24.242 & 2.194 & -2.371 & 24.303 & 2.152 & 0.024 & -0.061 & 0.043 \\
\hline 2 & -0.780 & 24.127 & 2.173 & -0.797 & 24.190 & 2.131 & 0.017 & -0.063 & 0.042 \\
\hline 3 & 1.805 & 23.952 & 2.122 & 1.800 & 24.017 & 2.082 & 0.005 & -0.065 & 0.040 \\
\hline 4 & -0.894 & 23.674 & 0.638 & -0.910 & 23.729 & 0.591 & 0.016 & -0.055 & 0.047 \\
\hline 5 & 1.417 & 23.434 & 0.570 & 1.412 & 23.491 & 0.525 & 0.005 & -0.057 & 0.045 \\
\hline 6 & -1.014 & 21.639 & 0.646 & -1.028 & 21.685 & 0.607 & 0.014 & -0.046 & 0.040 \\
\hline 7 & 0.740 & 21.784 & 0.591 & 0.734 & 21.832 & 0.552 & 0.006 & -0.048 & 0.040 \\
\hline 8 & 1.694 & 21.688 & 0.056 & 1.693 & 21.736 & 0.015 & 0.001 & -0.047 & 0.041 \\
\hline 9 & -1.149 & 19.545 & 0.642 & -1.160 & 19.581 & 0.610 & 0.012 & -0.036 & 0.032 \\
\hline 10 & 0.000 & 21.794 & 0.101 & -0.009 & 21.840 & 0.059 & 0.009 & -0.046 & 0.042 \\
\hline RMSE & & & & & & & 0.0124 & 0.0496 & 0.0390 \\
\hline
\end{tabular}

As could be seen from table 2, the standard deviations of $d X, d Y$ and $d Z$ were $0.0124 \mathrm{~m}, 0.0496 \mathrm{~m}$ and $0.0390 \mathrm{~m}$ respectively. Horizontal position error was $0.0511 \mathrm{~m}$ after calculation. The results of outdoor photogrammetry also can meet the requirements of 1:500 digital mapping.

By comparing these two experiments, it could be found that the error of experiment one was small and that of experiment two was large. The main reason was the shooting distance and the shooting environment. The measurement error would increase with the measurement distance. The complex environment would lead to measurement errors, such as unreasonable light was not conducive to the collection of images. This method could easily and quickly collect data outdoors. At the shooting distance of about 10 meters,
PhotoModeler Scanner could work out the three-dimensional coordinates of the target points and the measurement accuracy met the requirements of digital mapping.

\section{CONCLUSION}

In this paper, the USB camera is combined with the centering rod. Three pieces of image and three-dimensional coordinates of three shooting sites are taken as the basic calculation data, which are processed in PhotoModeler Scanner software. Finally, the three-dimensional coordinates of the target point are calculated and will be applied to detail survey. The experiment showed that when the shooting distance was 
about 10 meters, the error in the plane position of the target point was less than $10 \mathrm{~cm}$, and the accuracy met the requirements of detail survey. This method effectively solves the limitation of the object control points which close-range photogrammetry needed for, and makes full use of the advantage that close-range photogrammetry can carry out stereo measurement without contacting the target points. In addition, the equipment can reduce the field workload of detail survey, and reduce the volume, weight and cost of photographic equipment, thus it has certain application value. In order to improve the accuracy of stereo measurement, the system error is minimized when the equipment is installed.

\section{REFERENCE}

[1] Luhmann T. Close range photogrammetry for industrial applications[J]. Isprs Journal of Photogrammetry \& Remote Sensing, 2010, 65(6): 558-569.

[2] Yung-Chuan Chen, Yi-Hsing Tseng. Advancement of close range photogrammetry with a portable panoramic image mapping system (PPIMS)[J]. PHOTOGRAMMETRIC RECORD PHOTOGRAMMETRIC RECORD, 2018, Vol.33(162):196-216.

[3] HUANG Yun, DENG Fei, HU Yulei, et al. Application of Close-range Photogrammetry in the Dry Beach Monitoring[J]. Journal of Geomatics, 2016, 41(3):33-37.

[4] MAO Binbin, ZHANG Qiang, XU Hao, et al. Monitoring the Displacement and Deformation of the Slope of Hongqigou Dumping Site by Close-range Photogrammetry[J]. Modern Mining, 2016, 32(5):151-153.

[5] ZHANG Zuxun, ZHAN Zongqian, ZHENG Shunyi, et al. Photo Total Station System--The Integration of Digital Photogrammetry and Total Station[J]. Bulletin of Surveying and Mapping, 2005(11): 1-5.

[6] YANG Liu. Scheme Argumentation of Camera Transit Based on Non-Metric Digital Camera[D]. Xi'an: Xi'an University of Science and Technology, 2006.

[7] HE Yingpeng. Research on precision analysis and error correction based on Photo Total Station System[D]. Chongqing Chongqing Jiaotong University, 2016.

[8] GAO Shen, HU Qingwu, AI Mingyao, et al. Study of detail mapping method based on independent absolute orientation with GPS RTK and ordinary camera[J]. Science of Surveying and Mapping, 2017, 42(01): 107-112

[9] WANG Xinyu, FAN Baixing, YU Ying, et al. An automatic measurement method with visual guiding theodolite[J]. Engineering of Surveying and Mapping, 2018, 27(6):32-40.

[10] JU Xinghua. Implementation of non-control close-range photogrammetry system and its application[J]. Science of Surveying and Mapping, 2012, 37(3):188-190.

[11] CHENG Xiaojun, XU Chengquan ,ZHOU Xingquan. A Research on Rapid Calibration of Ordinary Digital Camera Based on PhotoModeler Scanner System[J]. Remote Sensing Information, 2011(4): 80-84.

[12] Zhang, Zhengyou. A flexible new technique for camera calibration[J]. IEEE Transactions on Pattern Analysis and Machine Intelligence, 2000, 22(11):1330-1334.

[13] HE X C, YUNG N H C. New method for overcoming ill-conditioning in vanishing-point-based camera calibration.[J] OPTICAL ENGINEERING, 2007, 46(3): 037202-037212.

[14] SONG Yuejun, HUANG Yanhe, YANG Jie, et al. Application of close-range photogrammetry technology in soil erosion monitoring $[\mathrm{J}]$. Science of Surveying and Mapping, 2016, 41(6): 80-83.

[15] FANG Ziyan, TANG Jianlin, DONG Xiangyong. Photogrammetry[M]. Wuhan: Changjiang Publishing House, 2012: 138-142. 